\title{
On undecidability of equicontinuity classification for cellular automata
}

\author{
Bruno Durand ${ }^{1}$ and Enrico Formenti ${ }^{1}$ and Georges Varouchas ${ }^{1}$ \\ ${ }^{1}$ Laboratoire d'Informatique Fondamentale de Marseille (LIF), \\ Centre de Mathématique et Informatique (CMI), 39 rue Joliot-Curie, 13453 Marseille Cedex 13, France. \\ email: $\{$ bdurand, eforment, gvarouch $\} e_{c m i}$.univ-mrs. fr
}

Equicontinuity classification is a popular classification of cellular automata based on their dynamical behavior. In this paper we prove that most of its classes are undecidable.

Keywords: cellular automata, classification, discrete dynamical systems, undecidability

\section{Introduction}

Cellular automata are simple model for the study of complex phenomena produced by simple local interactions. They consist of a regular lattice of cells. Each cell contain fi nite automaton which has a state chosen from a fi nite set of states. Updates are made according to a local rule which takes into account the current state of the cell and those of a fi xed fi nite set of its neighboring cells. All cells are updated synchronously. A snapshot of the state of all cells at the same time is called a configuration.

Despite of their defi nition simplicity, cellular automata exhibit a wide range of dynamical behaviors. The classifi cation of such behaviors is known as "the classifi cation problem". It has captivated researchers for years and a complete solution does not appear to be on coming.

The fi rst empirical classifi cation was proposed about twenty years ago by Wolfram after an extensive experimental work. Successive studies of researchers in the fi eld, tried to give this classifi cation a formal justifi cation. The fi rst attempts ořulik et al. [CY88, CPY89], made immediately clear were the problem is: CA behavior is so complex that almost any question about their long-term behavior is undecidable. Unfortunately we do not know how to formalize (and then prove) this idea. Two attempts in this direction are [Kar94] (a Rice theorem with variable states) and [CD00] (a partial Rice theorem with fi xed states).

In the middle of nineties, P. Kurka proposed to classify CA according to their degree of equicontinuity [Kur97]. Denote $E$ the set of equicontinuity points of a $\mathrm{CA}$ and $X$ the set of all confi gurations. Kurka devised the following four classes
K1) $E=X$;
$\left.K_{2}\right) E \neq \emptyset ;$
$\left.K_{3}\right) E=\emptyset$;
$\left.K_{4}\right)$ expansive CA .

1365-8050 (c) 2003 Discrete Mathematics and Theoretical Computer Science (DMTCS), Nancy, France 
Since many of these classes are defi ned by properties that are not strictly related to limit sets, one might suspect that at least some of them are decidable. In this paper we prove that the fi rst three classes are undecidable. These undecidability results are meaningful and have consequences on other decision problems (e.g. nilpotency). Decidability (or undecidability) of the fourth class is still open.

\section{Cellular automata, discrete dynamical systems}

Cellular automata are formally defi ned as quadruples $\langle d, Q, N, \delta\rangle$. The integer $d$ is the dimension of the space the cellular automaton works on. The set $Q$ is a fi nite set of states of cells. The neighborhood $N=\left(x_{1}, \ldots x_{v}\right)$ is a $v$-tuple of distinct vectors of $\mathbb{Z}^{d}$. The $x_{i}$ 's are the relative positions of the neighbor cells with respect to the cell, whose new state is being computed. The states of these neighbors are used to compute the new state of the center cell by the local function of the cellular automaton $\delta: Q^{v} \mapsto Q$.

A configuration is an application from $\mathbb{Z}^{d}$ to $Q$. Let $X=Q^{\mathbb{Z}^{d}}$ be the set of all confi guration. The local function induces a global function on $X$ as follows

$$
\forall c \in Q^{\mathbb{Z}^{d}}, \forall i \in \mathbb{Z}^{d}, \mathcal{A}(c)_{i}=\delta\left(c_{i+x_{1}}, \ldots, c_{i+x_{v}}\right)
$$

A state $q$ is called quiescent if $f(q, q, \ldots, q)=q$ (in the sequel we explain how our results are still valid when no quiescent state exists). More than one state can be quiescent, but only one of them is distinguished as the quiescent state. Denote $X_{F}$ the set of finite configurations, i.e., those confi gurations that are almost everywhere equal to the quiescent state — non-quiescent in a fi nite number of cells.

Let us now recall some classical defi nitions from discrete dynamical systems theory.

A dynamical system $(X, F)$ consists of a compact metric space $X$ and $F$ is a continuous function from $X$ to itself. Denote $d$ the metric on $X$ and $F^{n}$ the $n$-fold composition of $F$ with itself.

We are interested in the study of dynamical systems in symbolic spaces. $Q^{\mathbb{Z}^{d}}$ is endowed with the product topology (also called Cantor topology) of a countable product of discrete spaces on $Q$. For the sake of simplicity, we will study cellular automata in dimension $k=1$ or 2 . The generalization of results to higher dimensions is straightforward for all the results in the present paper.

The metric $d$ on $Q^{\mathbb{Z}^{d}}$ is defi ned as $\forall x, y \in Q^{\mathbb{Z}^{d}}, d(x, y)=2^{-n}$ where $n=\inf \left\{i \in \mathbb{N}, x_{i} \neq y_{i}\right.$ or $\left.x_{-i} \neq y_{-i}\right\}$. This metric induces the product topology on $Q^{\mathbb{Z}^{d}}$.

Denote by $Q^{\star}$ the set of fi nite words on $Q$. For $w \in Q^{\star},|w|$ denotes the length of $w$. For $t \in \mathbb{Z}, w \in Q^{\star}$, the sets

$$
[w]_{t}=\left\{x \in Q^{\mathbb{Z}}, x_{t}=w_{0}, \ldots, x_{t+|w|}=w_{|w|}\right\}
$$

are called cylinders and form a basis for the product topology on $Q^{\mathbb{Z}}$. The shift map $\sigma$ is often used as a paradigmatic example of chaotic symbolic system. It is defi ned as $\forall c \in Q^{\mathbb{Z}}, \forall i \in \mathbb{Z}, \sigma(c)_{i}=c_{i+1}$. Cellular automata are exactly those continuous maps from $Q^{\mathbb{Z}}$ to $Q^{\mathbb{Z}}$ that commute with the shift (i.e., $\mathcal{A} \circ \sigma=\sigma \circ \mathcal{A})$ [Hed69].

Kůrka's classes are defi ned by properties on the whole set of confi gurations. Using Proposition 1.1 one can express the properties defi ning some of Kưrka's classes in terms of behavior on fi nite patterns.

Proposition 1.1 ([Knu94]) Let $\mathcal{A}$ be a cellular automaton. Then $\mathcal{A}$ is sensitive to initial conditions w.r.t. finite configurations if and only if $\mathcal{A}$ is sensitive to initial conditions. 
A point $x \in$ is ultimately periodic if there exists two integers $\bar{t}, p(p>0)$ such that implies that $F^{p t+\bar{t}+i}(x)=$ $F^{\bar{t}+i}(x)$ for $i, t \in \mathbb{N}$. The minimal $\bar{t}, p$ with such a property are called, respectively, the length of the transient and the period of $x$. If $\bar{t}=0$ then $x$ is called periodic.

A point $x \in X$ is an equicontinuity point for $F$ if for any $\varepsilon>0$ there exists $\delta>0$ such that for any $y \in X$, $d(x, y) \leq \delta$ implies that $\forall t \in \mathbb{N}, d\left(F^{t}(x), F^{t}(y)\right)<\varepsilon$. If $X$ is perfect and all of its points are equicontinuity points then $F$ is equicontinuous.

In other words, an equicontinuous systems has a very stable dynamic. Small errors in initial conditions will not grow during evolutions. Periodic systems are a trivial example of equicontinous systems, although not all equicontinuos systems are periodic. In the special case of CA we have the following result.

Lemma 1.2 ([BT00]) A CA is equicontinuous if and only if it is ultimately periodic.

The notion of blocking word is rather intuitive central for characterizing cellular automata dynamics in the presence of equicontinuity points as we can see in Proposition 1.3. It will be crucial in the proof of the main result of this section.

A word $V \in Q^{\star}$ is blocking if there exists an infi nite sequence of words $(v)_{t \in \mathbb{N}}$ such that

1. for any $t \in \mathbb{N},\left|v_{t}\right|$ is fi nite, odd and greater than or equal to $r$;

2. for any $c \in[V]_{-(|V|-1) / 2}$ and any $t \in \mathbb{N}, A^{t}(c) \in\left[v_{t}\right]_{-\left(\left|v_{t}\right|-1\right) / 2}$.

In other words $V$ partitions the evolution diagram of $A$ in two completely disconnected parts: all perturbations made in one side are completely "blocked" by $V$.

Proposition 1.3 ([BT00]) Any equicontinuity point has an occurrence of a blocking word. Conversely, if there exist blocking words, then any point with infinitely many occurrences of a blocking word to the left and to the right (of 0 ) is an equicontinuity point.

Regularity is another property connected with periodicity. A system is regular if it has a dense set of periodic points.

A dynamical system $(X, F)$ is sensitive to initial conditions if there exists $\varepsilon>0$ such that for any $x \in X$ and any $\delta>0$, there exists $y \in X$ such that $0<d(x, y)<\delta$ implies that there exists $t \in \mathbb{N}$ such that $d\left(F^{t}(x), F^{t}(y)\right) \geq \varepsilon$. In other words a systems that is sensitive to initial conditions defeat all numerical simulations. In fact small error in the measure of initial confi gurations are magnifi ed during evolutions at a point that after some iterations the "real evolution" and the simulated one can be quite different.

Remark 1.4 In a perfect space, any system with no equicontinuity points is sensitive to initial conditions and vice-versa. This property, de facto, inspired the definition of Kürka's classes and separates class $K_{1} \cup K_{2}$ from $K_{3}$.

Expansivity is a stronger version of instability than sensibility to initial conditions (at least in perfect spaces). The system $(X, F)$ is expansive if there exists $\varepsilon>0$ such that for any $x, y \in X, d(x, y)>0$ implies that $\exists t \in \mathbb{N}$ such that $d\left(F^{t}(x), F^{t}(y)\right) \geq \varepsilon$. Be the defi nitions, it is easy to see that $K_{4}$ is contained in $K_{3}$.

\section{Undecidability of the class $K_{1}$}

Theorem 2.1 is the main result of this section. Its proof adresses the following problem in Kari's work [Kar94]: the set of states is not fi xed. The hardest part of the proof consists in proving that, in our particular case, letting the set of states vary is not necessary. 
Theorem 2.1 Consider $K_{1}$ for a fixed set of states $Q$ and a fixed dimension $d \in \mathbb{N}$. Then membership in $K_{1}$ is undecidable.

Remark 2.2 In [CY88], Culik and Yu prove that membership in $K_{1}$ is undecidable for one-dimensional $C A$ for any fixed set of states when considering finite configurations. This result cannot be used here since there are CA that are equicontinuous over finite configurations but not on more general configurations. Consider, for example, the one-dimensional CA on $\{0,1\}$ with local rule $\delta(x, y, z)=x y$ for $x, y, z \in\{0,1\}$.

In the proof of the main result of the section we make a reduction to a well-known undecidable problem for CA. To this purpose we need some more defi nitions and some results from literature.

A CA $\langle 1, Q, N, \delta\rangle$ is nilpotent if there exists an integer $n$ such that $\forall x \in Q^{\mathbb{Z}^{d}}, F^{n}(x)=\underline{c}$ for a quiescent confi guration $\underline{c}$. Given a CA $\langle 1, Q, N, \delta\rangle$, a state $q \in Q$ is spreading if $\delta\left(x_{1} \ldots x_{2} r+1\right)=q$ whenever there is at least one $i(1 \leq i \leq 2 r+1)$ such that $x_{i}=q$. Even if nilpotent CA (even with a spreading state) have a quite simple dynamics, these properties are undecidable as proved by the following result.

As pointed out by J. Kari and N. Ollinger [KO03], the proof technique of [Kar94] allows to assert the following result (stronger form than published in [Kar94] because of restriction to CA with a spreading states).

Theorem 2.3 Nilpotency is undecidable in the class of $1 D$ cellular automata with a spreading state.

However, Kůrka's classes are defi ned for a fi xed set of states, while the proof of the previous result does not allow to bound the cardinality of the set of states. We prove a slightly stronger version of this result:

Proposition 2.4 Nilpotency is undecidable for one-dimensional $C A$ with state set $\{0,1\}$.

Proof. Let $\mathcal{C} \mathcal{A}_{01 S}$ be the class of one-dimensional CA with state set $\{0,1\}$ such that $\delta\left(x_{-r} \ldots x_{r}\right)=0$ if $0^{r}$ is contained in $x_{-r} \ldots x_{r}$. For any confi guration $c$, call segment $w_{i, j}$ the word $w_{i, k}=c_{i+k}$ for $i \leq j$. Given a confi guration $c$, we will say that a segment $w_{i, n}$ is admissible if it does not contain the word $1^{n}$ and $w_{i-n, 2 n}$ does not contain $010^{n-1} 01$. Moreover we say that $c$ is $n$-admissible if any of its segment of length $n$ is admissible.

We reduce nilpotency to the problem of deciding nilpotency for CA in $\mathcal{C} \mathcal{A}_{01 S}$.

For any $\mathrm{CA} \mathcal{A}=\langle 1, Q,\{-r, \ldots, r\}, \delta\rangle$ with a spreading state, build the CA

$$
\mathcal{B}_{\mathcal{A}}=\left\langle 1,\{0,1\},\{-r n, \ldots, r n\}, \delta_{\mathcal{A}}\right\rangle
$$

where $n$ is the cardinality of $Q$. Let $I_{Q}=0,1, \ldots, n-1$ be a relabeling of the states of $\mathcal{A}$ such that 0 is a spreading state. We will code any state $q \in I_{Q}$ by $\pi(q)=01^{q} 0^{n-1-q}$.

For any $n$-admissible confi guration $c$, for any $i \in \mathbb{Z}$, denote $q(i)$ the state of the simulate cell. Remark that $q(i)$ can always be detected from $i$; in fact it suffi ces to fi nd the fi rst occurrence of 01 to the left of $i$. Now defi ne:

$$
\delta_{\mathcal{A}}\left(c_{i-r n}, \ldots, c_{i+r n}\right)=\pi(\delta(q(i-r), \ldots, q(i+r))) .
$$

By the above defi nition $\forall c \in Q^{\mathbb{Z}}, \mathcal{B}_{\mathcal{A}}(\pi(x))=\pi(F(x))$ where $F$ is the global function of $\mathcal{A}$. For all non $(2 r n+1)$-admissible segments, let

$$
\delta_{\mathcal{A}}\left(c_{i-r n}, \ldots, c_{i+r n}\right)=0 .
$$

Remark that since $\mathcal{A}$ is spreading then, by (2), $\mathcal{B}_{\mathfrak{A}}$ is $\mathcal{C} \mathcal{A}_{01 S}$. We claim that $\mathcal{A}$ is nilpotent if and only if $\mathcal{B}_{\mathfrak{A}}$ is nilpotent. 
Assume that $\mathcal{A}$ is nilpotent. Then there exists $n \in \mathbb{N}$ such that $F^{n}(c)=\underline{0}$. For all $n$-admissible confi gurations, by (1) and (2), we have that $F_{\mathcal{B}}^{n}(c)=\underline{0}$. If $c$ is not $n$-admissible, then let $i \in \mathbb{Z}$ be the index of a cell in a non-admissible segment. Remark that $0^{n}$ will occur in $F_{\mathcal{B}}(c)_{i-r n, 2 r n}$. Then since $\mathcal{B}_{\mathcal{A}}$ is in $C \mathcal{A}_{01 S}$ one fi nds $F_{\mathcal{B}}^{n}(c)_{i}=0$.

Assume that $\mathcal{A}$ is not nilpotent. By defi nition, there exists $c$ such for all $n \in \mathbb{N}, F^{n}(c) \neq \underline{0}$. Therefore, by (1), one fi nds that $\forall n \in \mathbb{N}, F_{\mathcal{B}}^{n}(\pi(c))=\pi\left(F^{n}(c)\right) \neq \underline{0}$. We thus get the required reduction.

The previous result can be generalized to arbitrary dimension and state set because 1-dimensional CA over $\{0,1\}$ are a decidable class among $d$-dimensional CA over $Q(\{0,1\} \subset Q)$, and analogously nilpotent cellular automata are decidable class among the class of ultimately periodic cellular automata.

Corollary 2.5 Nilpotency is undecidable for cellular automata on any fixed state set and dimension.

Theorem 2.6 Ultimate periodicity is undecidable among for CA on any fixed state set and dimension.

Proof of Theorem 2.1. Use Theorem 2.6 and Lemma 1.2.

\section{Undecidability of the class $K_{2}$}

Theorem 3.1 For a fixed dimension d and alphabet $Q$, it is undecidable if a CA on $Q^{\mathbb{Z}^{d}}$ has a blocking word.

We shall prove this result by proposing a reduction, inspired from [Sut89], of the classical Halting theorem to our problem. We will present this reduction in several steps: the fi rst step describes a universal class of Turing machines with constrains on their global behaviour, the second step will be the reduction of this particular universal model to cellular automata, and the fi nal step will prove that this last reduction can be done with a fi xed number of states.

By Proposition 1.3, the previous theorem and Remark 1.4, we get the following theorems.

Theorem 3.2 Given a set of states $Q$ and a dimension d, the class $K_{1} \cup K_{2}$ is undecidable on the class of CA over $Q^{\mathbb{Z}^{d}}$.

Theorem 3.3 Given a set of states $Q$ and a dimension d, the class $K_{3}$ is undecidable on the class of CA $\operatorname{over} Q^{\mathbb{Z}^{d}}$.

The reduction we propose is a reduction of the Halting problem on Turing machines to our $K_{2}$ problem over one-dimensional cellular automaton. The main diffi culty is that we want to enforce a fi xed behavior of our cellular automaton on all confi gurations, but not all confi gurations correspond to admissible instances.

There is a classical way to simulate a Turing machine $\mathcal{M}$ with a CA $C=\left\langle 1, Q_{C}, r=2, \delta\right\rangle$ : let $Q$ and $\Sigma$ denote the set of states and the working alphabet of $\mathcal{M}$, and $\mu$ its transition rule. We set $Q_{C}=Q \cup \Sigma$, and we defi ne $\delta$ as follows ( $*$ matches any state, and $\sigma$ any state in $\Sigma$ ):

- if $\mu\left(q_{1}, \sigma_{1}\right)=\left(q_{2}, \sigma_{2},+1\right)$, then $\delta\left(*, *, q_{1}, \sigma_{1}, *\right)=\sigma_{2}$ and $\delta\left(*, q_{1}, \sigma_{1}, *, *\right)=q_{2}$;

- if $\mu\left(q_{1}, \sigma_{1}\right)=\left(q_{2}, \sigma_{2}, 0\right)$, then $\delta\left(*, q_{1}, \sigma_{1}, *, *\right)=\sigma_{2}$ and $\delta\left(*, *, q_{1}, \sigma_{1}, *\right)=q_{2}$;

- if $\mu\left(q_{1}, \sigma_{1}\right)=\left(q_{2}, \sigma_{2},-1\right)$, then $\delta\left(*, q_{1}, \sigma_{1}, *, *\right)=\sigma_{2}, \delta\left(*, \sigma, q_{1}, \sigma_{1}, *\right)=\sigma$ and $\delta\left(*, *, \sigma, q_{1}, \sigma_{1}\right)=q_{2}$;

- for any unmatched neighborhood, $\delta$ does not modify the central cell. 
To represent the confi guration of $\mathcal{M}$ in the state $q$, with its reading head at position $i$ on the data string $w$, one can use the following fi nite confi guration ${ }^{\omega} 0 w_{[0 . . i-1]} q w_{[i .|w|]} 0^{\omega}$. One evolution step of $C$ on the above confi guration simulates one computation step of $\mathcal{M}$ on $w$.

Remark that this simulation does not allow us to link the properties of $\mathcal{M}$ with a behavior of $C$ on all its confi guration: the simulation is not consistent with $\mathcal{M}$ when $C$ acts on a confi guration which does not represent a confi guration of $\mathcal{M}$ (e.g. a confi guration with several reading heads, or an infi nite confi guration etc.).

A solution to this problem is suggested by Sutner in [Sut89]. He proposes a simulation process which constrains the behavior of the automaton on all fi nite confi gurations, and which we enhance to ensure properties over all possible confi gurations.

Instead of directly simulating the computation of the machine, we build an automaton $\mathcal{C}_{\mathcal{M}}$ which simulates a machine $\mathcal{P}_{\mathcal{M}}$ working on the following set of predicates: $\left\{P_{n}:=\right.$ "after $n$ computation steps starting on the empty entry $\varepsilon$, the machine $\mathcal{M}$ reaches the confi guration $w_{n}$ ", $\left.n \in \mathbb{N}\right\}$, where $w_{n}=\mathcal{M}^{n}(\varepsilon)$.

The fi rst step of our reduction will be the description of this machine $\mathcal{P}_{\mathcal{M}}$.

We represent $P_{n}$ with the string $\left[\bar{n} \mid w_{n}\right]$ ( $\bar{n}$ is the string representing $n$ in unary). On this representation, $\mathcal{P}_{\mathcal{M}}$ will be able to perform two things: compute $P_{n+1}$ from $P_{n}$ (i.e., simulate $\mathcal{M}$ 's computation), and also decide whether a given string represents a valid predicate or not (the set $\left\{P_{n} \mid n \in \mathbb{N}\right\}$ is recursive). In order to validate the behavior of $\mathcal{C}_{\mathscr{M}}$, we need to describe more precisely the behavior of $\mathcal{P}_{\mathcal{M}}$.

The data string of $\mathcal{P}_{\mathcal{M}}$ will have the following structure:

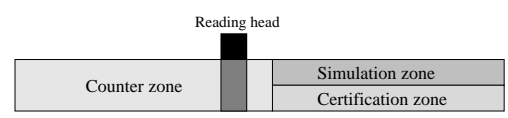

To implement such a structure, we use the alphabet $\Sigma_{\mathcal{P}}$ consisting in the union of the following sets:

- a singleton containing the blank character $B_{P}$;

- a set $\Sigma_{\text {count }}$ of two characters for the counter zone: $\begin{aligned} & 1 \\ & \square\end{aligned}$ and $\begin{aligned} & 1 \\ & \square\end{aligned}$ (both will be used to write $k$ in unary, and we need to be able to "check" each bit during the certifi cation cycle);

- a set $\Sigma_{\text {simul }}$ of characters for the simulation and certification zone:

$$
\Sigma_{\text {simul }}:=\left\{\begin{array}{l}
a \\
b
\end{array} \mid a, b \in \Sigma \cup Q\right\}
$$

(note that $\Sigma$ also contains the blank character of the machine $\mathcal{M}$ ). We shall distinguish two subsets: the set $\Sigma_{\text {char }}:=\Sigma \times(\Sigma \cup Q)$ (simulation of a character of $\mathcal{M}$ ) and the set $\Sigma_{\text {head }}:=Q \times(\Sigma \cup Q$ ) (simulation of the reading head of $\mathcal{M}$ );

- a set of separators, in order to point clearly out the various zones in the data string: [,| and ].

Call $I D$ (as in Instantaneous Description) the set of syntactically valid data strings for our machine $\mathcal{P}_{\mathcal{M}}$ :

$$
I D:=\left[\Sigma_{\text {count }}^{*} \mid \Sigma_{\text {char }}^{*} \Sigma_{\text {head }} \Sigma_{\text {char }}^{*}\right]
$$

The computation of $\mathcal{P}_{\mathcal{M}}$ simulating $\mathcal{M}$ on the empty entry proceeds as follows:

1. Check if the data string is in $I D$; 
2. simulate one computation step in the simulation zone (if necessary, extend the simulation zone by shifting the rightmost separator one cell to its right);

3. increment the counter by adding one extra $\begin{aligned} & 1 \\ & \square\end{aligned}$ to its left (thus extending the counter zone by one cell to its left);

4. uncheck the counter zone (i.e., replace all $\begin{array}{c}1 \\ \square\end{array}$ by $\left.\begin{array}{c}1 \\ \square\end{array}\right)$;

5. initialize the certifi cation zone (it should just contain the string describing $\mathcal{M}$ in its starting confi guration);

6. resimulate the $k$ fi rst steps of computation in the certifi cation zone, checking one bit in the counter zone at each step;

7. compare the result obtained in the certifi cation zone with the content of the simulation zone;

8. if no error occurred during the certifi cation, check whether the simulated machine $\mathcal{M}$ has reached a halting confi guration: if so, end the simulation (and enter an acceptance state), else proceed with the simulation loop.

During the simulation loop, several kind of errors can occur:

- during step $1, \mathcal{P}_{\mathcal{M}}$ can detect a syntax error;

- no error can occur during the steps 2 to 5 ;

- during step 6 , the certifi cation process should not use more space than delimited by the separators. The certifi cation can thus fail at this step if it appears that the space between the central and the rightmost separators is not enough;

- during step $7, \mathcal{P}_{\mathcal{M}}$ can detect a simulation error, if the simulation does not match the certifi cation;

- step 8 describes only the looping condition, and no error can appear here.

If any of these errors is raised, the machine enters a rejecting state, and halts.

Definition 3.4 (valid configuration) A configuration of $\mathcal{P}_{\mathcal{M}}$ is valid when the computation of $\mathcal{P}_{\mathcal{M}}$ on this configuration does not lead to a rejecting state.

We shall denote by $V_{\mathcal{M}}$ the set of valid confi gurations. $V_{\mathcal{M}}$ is recursive, and can be described as follows: a word $w$ is in $V_{\mathcal{M}}$ if and only if

- $w \in I D$

- if $k$ represents $w$ 's counter and $c$ its simulation zone, $c=\mathcal{M}^{k}(\varepsilon)$;

- the space between $w$ 's central separator and its rightmost separator is greater than or equal to the number of cells used by $\mathcal{M}$ during its $k$ fi rst computation steps.

The following remarks will be useful in the sequel.

Remark 3.5 One whole simulation loop simulates exactly one computation step of $\mathcal{M}$.

Remark 3.6 By the certification process it holds that: $\mathcal{P}_{\mathcal{M}}$ will complete a simulation loop without entering a rejecting state if and only if its data string represents a predicate in $V_{\mathcal{M}}$.

Remark 3.7 Only Step 2 and 3 allow $\mathcal{P}_{\mathcal{M}}$ to use extra cells on its data string. Thus, if $\mathcal{P}_{\mathcal{M}}$ completes a simulation loop, the length of the data string increase by at most 1 to its right, and exactly 1 to its left. 
Denote by $Q_{P}$ the set of states of $\mathcal{P}_{\mathcal{M}}$.

Lemma 3.8 The three following statements hold:

1. $\mathcal{M}$ halts on the empty entry if and only if $\mathcal{P}_{\mathcal{M}}$ halts on all entries.

2. If $\mathcal{M}$ halts in $k$ steps on $\varepsilon$, then, on any entry of size $s, \mathcal{P}_{\mathcal{M}}$ halts and uses at most $s+2 k$ cells during its computation.

3. If $\mathcal{M}$ doesn't halt on $\varepsilon$, then there is an entry of $\mathcal{P}_{\mathcal{M}}$ on which $\mathcal{P}_{\mathcal{M}}$ doesn't halt and uses an unbound number of cells during its computation.

Proof. Consider an entry $w$ of $\mathcal{P}_{\mathcal{M}}$.

1. If $w \notin V_{\mathcal{M}}$, then by Remark 3.6, we know that $\mathcal{P}_{\mathcal{M}}$ halts on $w$.

If $w \in V_{\mathscr{M}}$ then

- either $\mathcal{M}$ halts on $\varepsilon$, then any computation on a confi guration which derives from $\varepsilon$ leads to termination, hence the computation of $\mathcal{P}_{\mathcal{M}}$ on $w$ eventually terminates;

- or $\mathcal{M}$ does not halt on $\varepsilon$; then, by Remark $3.5, \mathcal{P}_{\mathcal{M}}$ will compute indefi nitely many simulation loops on $w$, and will not stop.

2. By Remark 3.5, if $\mathcal{M}$ halts on $\varepsilon$ in $k$ steps, then any computation of $\mathcal{P}_{\mathcal{M}}$ involves at most $k$ simulation loops, and therefore, by Remark 3.7, the growth of the data string is bounded by $2 k$.

3. If $\mathcal{M}$ does not halt on $\varepsilon$, then, by Remark 3.5, $\mathcal{P}_{\mathcal{M}}$ 's computation on $\left.\left[\mid \begin{array}{c}q_{0} \\ -\end{array}\right]\right]$ does not halt too.

The simulation loop is thus executed an unbounded number of times, and by Remark 3.7, one fi nds that after each simulation loop, the data string grows by at least one cell.

Let us now describe the second step of our reduction: $\mathcal{C}_{\mathcal{M}}$ will mainly consist in a classical simulation of $\mathcal{P}_{\mathcal{M}}$, but it will be able to erase the zones of its confi gurations which do not correspond to a confi guration of $\mathcal{P}_{\mathcal{M}}$.

More precisely: we set $Q_{\mathcal{C}_{\mathcal{M}}}:=\Sigma_{\mathcal{P}} \cup Q_{\mathcal{P}} \cup\{$ kill $\}$. These states will play different roles in the sequel;

- 0 denotes the blank character $B_{P}$;

- $Q_{\text {inert }}$ denotes the subset $\Sigma_{P}-\left\{B_{P}\right\}$;

- $Q_{\text {head }}$ denotes the subset $Q_{\mathcal{P}}$.

The radius is 4 , and the local rule acts as follows:

1. if a cell is in the state $\mathrm{kill}$, it enters the state 0 ;

2. if a cell's state is neither kill nor 0 , and there is at least a cell kill state in its neighborhood, it enters the kill state;

3. if a cell's state is neither kill nor 0 , and there is at a local configuration error in its neighborhood, it enters the kill state;

4. otherwise, the usual simulation rule of a Turing machine by a cellular automaton is applied.

A local configuration error on a given confi guration is a local block (of radius 4) of the confi guration which should not appear in a valid simulation. More precisely, we will have a local confi guration error when the block contains: 
- $\mathcal{P}_{\mathcal{M}}$ 's rejecting state,

- non blank characters to the right of a right separator ],

- non blank characters to the left of a left separator [,

- two reading heads, that is two cells whose states are in $Q_{\text {head }}$.

Definition 3.9 (data zone, active signal) Given a configuration c, a data zone on this configuration will be any maximal connected part of $c$ which does not contain 0.

With the local rule of $\mathcal{C}_{\mathcal{M}}$ defined above, we can see that the only possible modifications can occur around cells with a state in $Q_{\text {head }} \cup\{\mathrm{kill}\}$. We will call these states active signals.

A confi guration of $C_{\mathcal{M}}$ can be seen as a sequence of data zones, on which $\mathcal{C}_{\mathcal{M}}$ acts through these active signals. The kill signal is invoked whenever a simulation error is detected, and destroys the zone in which it appears; the other signals (which represent the trajectory of $\mathcal{P}_{\mathcal{M}}$ 's reading head) modify the data zone, possibly extending it.

Lemma 3.10 If the kil 1 state appears inside a finite data zone, this data zone will be entirely erased after a finite number of steps.

Proof. With the radius of our automaton, the $\mathrm{kill}$ signal is at least twice as fast as the other active signals: it can act on a neighborhood of radius 4 , while the other signals, following the classical simulation rule, act on a neighborhood of radius 2. Thus, if a kill signal appears in a fi nite data zone, the other signals will not be able to compensate indefi nitely its destruction.

Definition 3.11 (valid zone) A data zone will be valid when it represents a real valid configuration of $\mathcal{P}_{\mathcal{M}}$, that is a word in $V_{\mathcal{M}}$, with an adequately placed reading head.

In other words: a valid data zone is a fi nite zone, which represents a real simulation of $\mathcal{P}_{\mathcal{M}}$, in which no kill signal will be invoked, unless it interacts with other data zones on the confi guration.

Lemma 3.12 The two following hold:

1. if a data zone is not valid, it will grow by at most 2 cells;

2. if a data zone is valid, it will grow, for each complete loop simulated by $\mathcal{C}_{\mathcal{M}}$ in this zone, by exactly one cell to the left and at most one to the right.

Proof. A data zone can only grow under the action of a signal in $Q_{\text {bead }}$. Hence, if the invalid data zone does not contain a reading head character, it will not grow.

Else, if it is not valid, at least one kill signal will be invoked before one complete loop of $\mathcal{P}_{\mathcal{M}}$ could be simulated by $\mathcal{C}_{\mathcal{M}}$ in this data zone. Steps 2 and 3 of $\mathcal{P}_{\mathcal{M}}$ 's loop will therefore be simulated at most once each, and the data zone will grow by at most 2 cells.

Statement 2 follows from the simulation process of $\mathcal{P}_{\mathcal{M}}$ by $\mathcal{C}_{\mathcal{M}}$, and Remark 3.7.

Lemma 3.13 If $\mathcal{M}$ halts on the empty entry in $k$ steps, then $0^{2 k+5}$ is a blocking word of $\mathcal{C}_{\mathfrak{M}}$.

Proof. By Lemma 3.12 that, if $\mathcal{M}$ only computes $k$ steps on $\varepsilon$, then the growth of any data zone is bounded by $k$ cells to the left and $k$ to the right.

If the word $0^{2 k+5}$ is present in the confi guration, it defi nes (at least) two data zones (one to its left and one to its right), which won't grow more than $k$ cells to either side along the evolution of $\mathcal{C}_{\mathcal{M}}$. Hence the fi ve central cells of the word $0^{2 k+5}$ will never be affected, which ends the proof. 
Lemma 3.14 If $\mathcal{M}$ does not halt on the empty entry, then no word of $Q^{*}$ is blocking for $\mathcal{C}_{\mathcal{M}}$.

Proof. Consider a word $w$ of $Q^{*}$, we want to prove that we can always create around $w$ a confi guration which disturbs any letter of $w$. By Lemma 3.12, any valid data zone will grow indefi nitely to its left, unless it interacts with another data zone.

Consider a word $v$ representing a valid zone. If we let $\mathcal{C}_{\mathcal{M}}$ evolve on the confi guration $\varphi_{0}:={ }^{\omega} 0 w v 0^{\omega}$, then exactly one of the following statements holds:

- all the data zones of $w$ disappear completely before the evolution of $v$ gets a chance to interact with it;

- $v$ 's evolution interacts with some data zone of $w$.

If the first case holds, then $w$ is not a blocking word: $v$ 's evolution can proceed indefi nitely, and will eventually reach any cell to its left.

If the second case holds: a kill signal is invoked after a fi nite number of steps, in the middle of a fi nite data zone (the concatenation of $w$ 's rightmost data zone and $v$ 's evolution), so by Lemma 3.10, this data zone will be erased in a fi nite number of steps $n_{1}$.

Now, consider the evolution of the confi guration $c_{1}:={ }^{\omega} 0 w v 0^{2 n_{1}} v 0^{\omega}$.

We know that the fi rst $v$ will disappear along with $w$ 's rightmost data zone after $n_{1}$ steps. The space between the two $v$ makes it thus impossible for the second to interact with the fi rst. Therefore examining the evolution of $\mathcal{C}_{\mathfrak{M}}$ on this confi guration, exactly one of two statements holds:

- all the data zones of $w v$ disappear completely before the evolution of the second $v$ gets a chance to interact with it;

- the second $v$ 's evolution interacts with some data zone of $w$.

If the first case holds, $w$ is not a blocking word.

In the second case: another data zone of $w$ will be destroyed, after a certain number of steps $n_{2}$. In this case, we can consider the confi guration $c_{2}:={ }^{\omega} 0 w v 00^{2 n_{1}} v 0^{2 n_{2}} v 0^{\omega}$.

By induction, we can thus build an arbitrarily long sequence of confi gurations $\left(c_{k}\right)$, such that the confi guration $q_{k}$ "kills" $k$ data zones of $w$. Since $w$ is fi nite, it only has a fi nite number of data zones, and eventually disappears entirely.

Corollary 3.15 $\mathcal{C}_{\mathcal{M}}$ has an equicontinuity point if and only if $\mathcal{M}$ halts on $\varepsilon$.

Proof of Theorem 3.2. We will now describe the fi nal part of our reduction: we need to prove that the reduction $\mathcal{M} \rightarrow C_{\mathscr{M}}$ can be done even if the set of states of $\mathcal{C}_{\mathscr{M}}$ is fi xed. As in the preceding section, we will build a similar reduction with a 2 -state automaton.

From $\mathcal{C}_{\mathcal{M}}$ build the 2-state automaton $\widetilde{C_{\mathcal{M}}}$ which simulates $\mathcal{C}_{\mathcal{M}}$ as follows: choose a fi xed indexation of $\mathcal{C}_{\mathcal{M}}$ 's states $Q=\left\{q_{0}, \ldots, q_{n}\right\}$, imposing $q_{0}=0$, and represent $q_{i}$ by the binary sequence $01^{i} 0^{n-i}$.

A state of $\mathcal{C}_{\mathscr{M}}$ is thus represented by a sequence of $n+1$ states of $\widetilde{C_{\mathcal{M}}}$. The radius of $\mathcal{C}_{\mathcal{M}}$ is 4 , so a radius of $5(n+1)$ for $\widetilde{\mathcal{C}_{\mathcal{M}}}$ is enough for a cell to determine both the state to which it belongs, and the state of its two simulated neighbors.

The beginning of a simulated state is represented by the sequence 01 , so that any cell of a simulated state is able to determine if, locally, the simulated confi guration is valid (there must be exactly a multiple of $(n+1)$ cells between two consecutive occurrences of 01$)$. 
The local rule of $\widetilde{\mathcal{C}_{\mathcal{M}}}$ proceeds as follows: if the neighborhood of a cell contains an alignment error, the cell enters the state 0 , else the cell evolves according to the simulated rule.

Both Lemma 3.13 and 3.14 can be adapted to $\overline{\mathcal{C}_{\mathcal{M}}}$ : the simulation of $0^{2 k+5}$, that is $0^{(2 k+5)(n+1)}$, is a blocking word for $\widetilde{C_{\mathcal{M}}}$ if $\mathcal{M}$ halts on $\varepsilon$; and if $\mathcal{M}$ does not halt, then any simulated valid zone will also grow indefi nitely, allowing to perturb any given fi nite word.

\section{Acknowledgements}

The authors are very grateful to Jarkko Kari and Nicolas Ollinger for pointing out the result about nilpotent CA with a spreading state that was fundamental to extend our proof in Section 2 (originally designed for dimension at least 2) to all dimensions.

\section{References}

[BT00] F. Blanchard and P. Tisseur. Some properties of cellular automata with equicontinuity points. Annales de l'Instute Henri Poincaré, 36(5):562-582, 2000.

[CD00] J. Cervelle and B. Durand. Tilings: Recursivity and regularity. In STACS 2000, volume 1770 of $L N C S$, pages 491-501, 2000.

[CPY89] K. Culik, J. Pachl, and S. Yu. On the limit set of cellular automata. SIAM Journal on Computing, 18:831-842, 1989.

[CY88] K. Culik and S. Yu. Undecidability of cellular automata classifi cation schemes. Complex Systems, 2:177-190, 1988.

[Hed69] G. A. Hedlund. Endomorphism and automorphism of the shift dynamical system. Mathematical System Theory, 3:320-375, 1969.

[Kar94] J. Kari. Rice's theorem for the limit set of cellular automata. Theoretical Computer Science, 127:229-254, 1994.

[Knu94] C. Knudsen. Chaos without nonperiodicity. American Mathematical Montly, 101:563-565, 1994.

[KO03] J. Kari and N. Ollinger. Why proof of indecidability of nilpotency applies to cellular automata with a spreading state. Personal communication, 2003.

[Kur97] P. Kurka. Languages, equicontinuity and attractors in cellular automata. Ergodic Theory \& Dynamical Systems, 17:417-433, 1997.

[Sut89] K. Sutner. A note on culik-yu classes. Complex Systems, 3:107-115, 1989. 
